\title{
Formation and Structure of Human Hageman Factor
}

\section{Fragments}

\author{
Joseph T. Dunn and Allen P. Kaplan, Division of Allergy, Rheumatology, and \\ Clinical Immunology, Department of Medicine, State University of New \\ York, Stony Brook, New York 11794
}

A B S T R A C T Autodigestion of activated Hageman factor $(\mathrm{HFa})$ yields a 40,000-mol wt activated enzyme as well as Hageman factor fragment (HFf); HFf consists of two molecular weight species of 28,500 and 30,000 . We have investigated the structure of these active fragments and demonstrate that upon reduction, each possesses a heavy chain of 28,000 . The associated light chains were identified by subjecting iodinated proteins to two-dimensional slab gel electrophoresis in which the second dimension is run reduced. The 40,000-dalton enzyme has a light chain of 15,000, the 30,000-dalton form of HFf has a light chain of 2,000 and we have suggestive evidence of a light chain associated with the 28,500-dalton form of HFf (putative mol wt $\sim 500$ ). We also demonstrate that the 30,000 -dalton form of HFf precedes the 28,500 form. These data indicate that digestion of native HF to form $\mathrm{HFa}$ precedes cleavages that fragment the molecule and diminish its molecular weight. The 28,500-dalton light chain of $\mathrm{HFa}$ becomes the heavy chain of each of the fragmentation products while cleavage at different points along the heavy chain of HFa determines which fragments will be produced. In contrast to autoactivation, kallikrein digestion of $\mathrm{HFa}$ yields primarily HFf; however, the 40,000-dalton enzyme may be seen when prekallikrein-deficient (Fletcher trait) plasma is activated.

\section{INTRODUCTION}

We have previously shown that cleavage of Hageman factor $(\mathrm{HF})^{1}$ by activated Hageman factor $(\mathrm{HFa})$, (autodigestion) (1) or by kallikrein first yields a two-chain

Received for publication 26 February 1982 and in revised form 5 May 1982.

${ }^{1}$ Abbreviations used in this paper: DNS-GGACMK, dansyl-glutamic acid-glycine-arginine-chloro-methyl ketone; HF, Hageman factor; HFa, activated Hageman factor; HFf, Hageman factor fragment; 2-ME, 2-mercaptoethanol. enzyme (HFa) in which a heavy chain of mol wt 52,000 is disulfide linked to a light chain of 28,000 (2). This form of activated HF, which has the same molecular weight as the native molecule, was first demonstrated functionally by Kaplan and Austen (3) and its structure described by Revak et al. (2). Further digestion of HFa by kallikrein (4) yields Hageman factor fragment (HFf) that consists of two molecular weight forms of $\sim 28,000-30,000$ (5) each of which has an active site (1). Autodigestion of $\mathrm{HFa}$, however, produces not only HFf, but also an active 40,000-mol wt fragment (1) corresponding to one of the intermediate-sized enzymes reported (3).

In this study we have further investigated the structure of HFf as well as the 40,000 -dalton moiety and demonstrate that upon reduction, each possesses a heavy chain of 28,000 . The 40,000 -dalton enzyme has a light chain of 15,000 , whereas the 30,000 -dalton form of HFf has a light chain of 2,000 . We also demonstrate that the 30,000-dalton form of HFf precedes the formation of a 28,500-dalton form and suggest that the latter molecule possesses a 500-dalton light chain. Our data support the conclusion that $\mathrm{HFa}$ formation precedes formation of any of the activated forms of $\mathrm{HF}$ $(1,3)$ and demonstrate that HFf is a two-chain enzyme. The implications of these conclusions upon the mechanism of HF activation and the sites at which cleavage occurs are discussed.

\section{METHODS}

Chemicals used in the purification of HF were obtained from sources used previously (6). Other reagents were: H-D-proline-phenylalanine-arginine pNA (H-D-Pro-Phe-Arg pNA) (S2302) (Kabi Group, Inc., Greenwich, CT); ultra pure urea and chymotrypsinogen (Schwartz-Mann Div., Becton, Dickinson \& Co., Orangeburg, NY); transferrin, sodium dodecyl sulfate (SDS), tris base, dextran sulfate $(M, 500,000)$, benzamidine, and 2-mercaptoethanol (2-ME) (Sigma Chemical Co., St. Louis, MO); ${ }^{125} \mathrm{I}$ Bolton-Hunter reagent and $\mathrm{Na}{ }^{125} \mathrm{I}$ (New England Nuclear, Boston, MA); Kodak XR-5, X-ray film, Tri-X $4 \times 5$ negative film, and Kodak lanex intensifying 
screens (Eastman Kodak Co., Rochester, NY); and reagents for electrophoresis and lactoperoxidase/glucose oxidase solid phase enzymobeads (Bio-Rad Laboratories, Richmond, CA). Dansyl-glutamic acid-glycine-arginine-chloro-methyl ketone (DNS-GGACMK) was a generous gift from Dr. Eliot Shaw and Dr. Charles Kettner of Brookhaven National Laboratories, Upton, NY

HF (6), HFf, (6), and prekallikrein (7) were purified from human plasma by previously published procedures. Kallikrein was prepared by activation of prekallikrein by $\mathrm{HFf}$ and isolated by passage over QAE Sephadex (6). All plasma proteins were dialyzed into $10 \mathrm{mM}$ sodium phosphate, $\mathrm{pH}$ 7.8 , and 0.15 sodium chloride before use. Protein concentrations were determined using the method of Lowry et al. (8) calibrated with human immunoglobulin G (IgG). The concentration of the IgG standard was determined spectrophotometrically using the value $\mathrm{E}_{280}^{0.1 \%}=1.42$. The assay gave a linear calibration for the range of 1 to $25 \mu \mathrm{g}$ of protein in a 200- $\mu$ l sample. DNS-GGACMK was dissolved in $0.001 \mathrm{~N}$ $\mathrm{HCl}$ at $1 \mathrm{mM}$ and appropriate dilutions were made for addition to protein solutions.

Polyacrylamide gel electrophoresis in the presence of SDS was performed according to the method of Laemmli (9), using the tube gels or vertical slab gels. The acrylamide concentration was $3.5 \%$ in the spacer gel and $10 \%$ in the separating gel. The samples were prepared for electrophoresis in Laemmli's sample buffer, which contained the final concentrations of 0.0625 Tris- $\mathrm{HCl}$ ( $\mathrm{pH} \mathrm{6.8),} \mathrm{2 \%} \mathrm{SDS,} \mathrm{10 \%}$ glycerol, and $0.001 \%$ bromophenol blue as the dye. After electrophoresis gels were fixed in $10 \%$ acetic acid and $25 \%$ isopropanol for periods varying from $15 \mathrm{~min}$ to overnight and dried for autoradiography or stained with Coomassie Brilliant Blue R-250 (Eastman Kodak Co.). Molecular weights were determined by running proteins of known molecular weight on gels with the samples.

For identification of active site generation, native $\mathrm{HF}$ $(15.6 \mu \mathrm{M})$ was incubated with $50 \mu \mathrm{g} / \mathrm{ml}$ dextran sulfate and kallikrein $(0.42 \mu \mathrm{M})$ in a total volume of $128 \mu \mathrm{l}$ at $37^{\circ} \mathrm{C}$. At timed intervals, $12 \mu \mathrm{l}$ of the HF-kallikrein-dextran sulfate mixture was removed and made $165 \mu \mathrm{M}$ in DNS-GGACMK and incubated for $30 \mathrm{~min}$ at $37^{\circ} \mathrm{C}$.

Sample buffer was then added with and without $2 \mu$ l of 2-mercaptoethanol and the samples were heated for $2 \mathrm{~min}$ at $100^{\circ} \mathrm{C}$. After SDS slab gel electrophoresis the gels were transilluminated on a long wave ultraviolet light box. Photographs were made using a red filter and either Polaroid type $57 /$ high speed or Kodak 4177 high speed $4 \times 5$ film. After photography the gels were stained and dried.

Samples used in two-dimensional gel electrophoresis to determine the structure of HFf and the active species with a mol wt of 40,000 were iodinated using Bolton-Hunter reagent. A sample of $0.6 \mathrm{ml}$ having a protein concentration of $0.21 \mathrm{mg} / \mathrm{ml}$ containing HFf and active 40,000 dalton species was made $1 \%$ in SDS by the addition of $66 \mu \mathrm{l}$ of a $10 \%$ SDS solution and placed in a boiling water bath for $3 \mathrm{~min}$. The mixture was added to a glass combi-V-vial (New England Nuclear) in which $250 \mu \mathrm{Ci}$ of Bolton-Hunter reagent had been dried with nitrogen gas. The mixture was incubated for $16 \mathrm{~h}$ at room temperature and dialyzed exhaustively in $10 \mathrm{mM}$ sodium phosphate buffer pH 7.8 containing $0.1 \mathrm{M}$ $\mathrm{NaCl}$ and $0.005 \%$ Triton $\mathrm{X}-100$. Two $15 \%$ SDS tube gels $(0.4$ $\times 11.5 \mathrm{~cm})$ were loaded with $15 \mu \mathrm{l}$ of the sample $\left(2.5 \times 10^{5}\right.$ $\mathrm{cpm} / \mu \mathrm{g}$ ) and electrophoresed as described above.

The second dimension consisted of a linear acrylamide gradient ranging from 12 to $20 \%$, constructed using a Buchler Universal Mixer, Buchler Instruments, Fort Lee, NJ, with a vibration stirrer attached to a Gilson Minipuls 2 peristaltic pump (Gilson Medical Electronics, Middleton, WI). $11.5 \mathrm{ml}$ of $12 \%$ and $20 \%$ acrylamide in $0.375 \mathrm{M}$ Tris- $\mathrm{HCl}, \mathrm{pH} 8.8$, containing $0.1 \%$ SDS and $0.15 \% N, N, N^{\prime}, N^{\prime}$,tetramethyl ethylenediamine were made $0.025 \%$ with ammonium persulfate, pumped into the glass form, and allowed to polymerize. A stacking layer of $3 \%$ agarose in $0.125 \mathrm{M}$ Tris- $\mathrm{HCl}, \mathrm{pH} 6.8$, containing $0.1 \%$ SDS with or without $0.5 \% 2-\mathrm{ME}$ was layered on top. The first dimension tube gel $(0.4 \times 11.5 \mathrm{~cm})$ containing the protein sample was embedded on the stacking layer by overlaying it with the hot agarose solution. Low molecular weight prestained marker proteins (Bethesda Research Labs, Bethesda, MD) were placed onto Whatman 1 filter paper (Whatman Inc., Paper Div., Clifton, NJ) and embedded adjacent to the first dimension gel.

\section{RESULTS}

To assess the kinetics of HFf formation a time course of $\mathrm{HF}$ cleavage by kallikrein was performed in the presence of macromolecular dextran sulfate as an initiating surface, and the active enzyme forms produced were labeled with the dansylated active site inhibitor DNS-GGACMK. The fluorescent pattern resulting is shown in Fig. 1. By $1 \mathrm{~min}$, most of the fluorescence is seen at mol wt 80,000 , indicating $\mathrm{HFa}$ formation and a trace of fluorescence at 30,000 daltons is also seen. With time the 30,000 -dalton band increases in intensity up to $45 \mathrm{~min}$ and then decreases. A 28,500 dalton enzyme becomes visible at $\sim 5 \mathrm{~min}$ and continues to increase throughout the time course. During

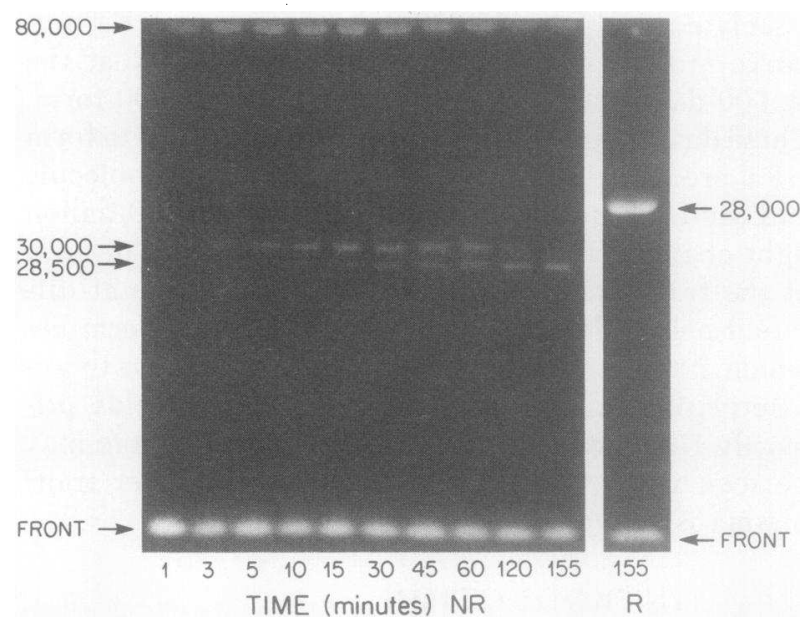

Figure 1 Visualization of the active forms of $\mathrm{HF}$ after cleavage by kallikrein using the active site inhibitor DNSGGACMK. HF was incubated with dextran sulfate and kallikrein for the time indicated. Aliquots were removed, treated with DNS-GGACMK, and electrophoresed under nonreducing conditions. The visible fluorescent bands run at positions that correspond to molecular weights of 80,000 , 30,000 , and 28,500 . The $155-\mathrm{min}$ aliquot was also electrophoresed under reducing conditions and runs as a single band of fluorescence with a molecular weight of 28,000 . The lowest bands are unincorporated DNS-GGACMK running at the dye front. 
this period $\mathrm{HFa}$ remains constant to $\sim 30 \mathrm{~min}$ and then is depleted. Upon reduction of the mixture at $155 \mathrm{~min}$, all of the active sites are found at mol wt of 28,000 . This suggests that $\mathrm{HFa}$ and the two molecular weight forms of HFf have in common a 28,000-dalton chain that possesses the reactive enzymatic site.

We next digested HF with kallikrein in the fluid phase and isolated the active fragments by QAE Sephadex chromatography as described (6). Fig. 2 shows nonreduced SDS gels of a mixture of the active fragments of mol wt $40,000,30,000$, and 28,500 . Upon reduction, only a $28,000-\mathrm{mol} \mathrm{wt}$ band is seen. This pattern of cleavage is seen upon autodigestion of $\mathrm{HF}$ upon surfaces (1) or by fluid phase digestion by enzymes such as kallikrein or plasmin $(2,3)$. Because the molecular weight of the nonreduced mixture was $>28,000$ we anticipated seeing low molecular weight bands upon reduction, but did not. We therefore radiolabeled the mixture with ${ }^{125}$ I using Bolton-Hunter

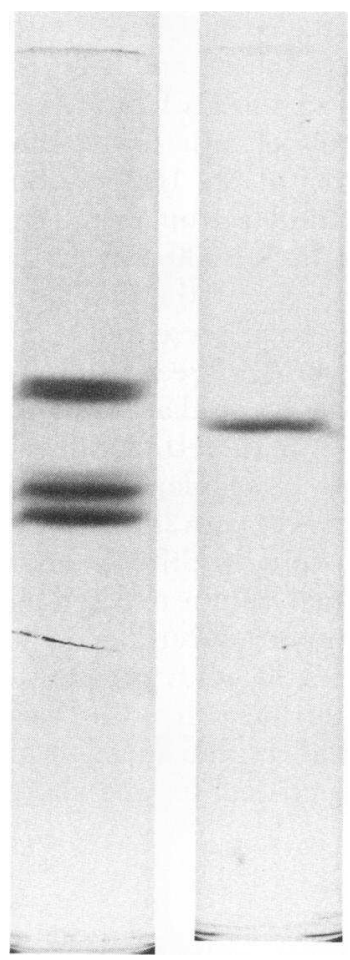

FIGURE 2 SDS gel electrophoresis of a sample containing both HFf and the active species having a mol wt of 40,000 . Samples were electrophoresed under nonreducing conditions (left). The upper band has a mol wt of 40,000 and the doublet below has mol wt of 30,000 and 28,500 (HFf). When electrophoresed under reducing conditions (right) a single species is seen having a mol wt of 28,000 . Note: samples run under reducing conditions (as well as the molecular weight standards) migrate with decreased mobility when compared to nonreduced samples.
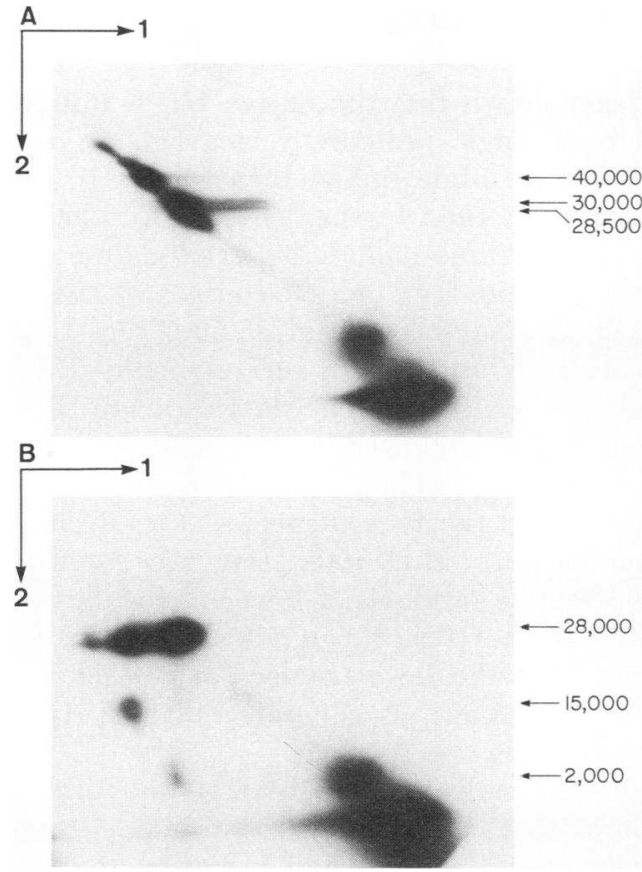

FIGURE 3 Two-dimensional electrophoresis of ${ }^{125}$ I HFf and the ${ }^{125}$ I 40,000-dalton enzyme labeled using the BoltonHunter reagent. ${ }^{125}$ I labeled proteins in $15 \%$ SDS polyacrylamide tube gels under nonreducing conditions, and electrophoresed vertically into the second dimension through (A) a 3\% agarose layer or (B) a 3\% agarose layer having $0.5 \%$ 2-ME into an SDS slab gel made up of an acrylamide gradient of 12 to $20 \%$ as described in Methods. Molecular weights were determined by running prestained low molecular weight markers of ovalbumin $(43,000), \alpha$-chymotrypsinogen $(25,700)$, cytochrome $(12,300), \quad \beta$-lactoglobulin $(18,400)$, lysozome $(14,300)$, bovine trypsin inhibitor $(6,200)$, and insulin ( $\mathrm{A}$ and $\mathrm{B}$ chains) $(\sim 3,000)$ into the second dimension.

reagent and then examined the material by two-dimensional gel electrophoresis and radioautography. Fig. 3A shows the pattern obtained when HFf and the 40,000-dalton enzyme are run nonreduced in both dimensions. The labeled species are visible along the diagonal. In Fig. 3B, the iodinated species are run nonreduced in the first dimension and then reduced in the second dimension. The active species at mol wt 40,000 generates bands with apparent mol wt of 28,000 and 15,000 . HFf appears as bands with mol wt of 28,000 and 2,000. A trace additional band is seen at the leading electrophoretic edge. Thus each of these enzymes appears to consist of a 28,000-dalton heavy chain to which a light chain of varying molecular weight is disulfide linked. These low molecular weight light chains apparently do not stain well with Coomassie Blue and are readily missed when gels are stained and inspected visually. 


\section{DISCUSSION}

It has been shown that the native HF is initially activated by a single proteolytic cleavage to yield the two-chained disulfide linked $\mathrm{HFa}$ form $(10,11)$ and that the $\mathrm{HFa}$ is then further cleaved to other active species $(1,3)$. These include a 40,000-dalton enzyme molecule (1) and HFf, which consists of two active species of mol wt 30,000 and 28,500. Fig. 1 depicts active site generation when surface-bound HF is incubated with kallikrein. The data indicate that $\mathrm{HFa}$ is formed initially followed by generation of the 30,000 mol wt form of HFf. The 30,000-dalton form is further cleaved to yield the 28,500 species. This is consistent with our earlier results indicating a sequential formation of active products of lower molecular weights generated from $\mathrm{HFa}(2)$; and demonstrates, for the first time, that the lower molecular weight form of HFf (mol wt 28,500) is generated directly from the higher molecular weight form.

Because the molecular weight of HFf is within 2,000 to 3,000 of that of the light chain obtained upon reduction of $\mathrm{HFa}$, it has been proposed that $\mathrm{HFf}$ is formed by cleavage outside of a small disulfide bridge. Because HFf is seen as an active doublet, this cleavage must occur at two sites. Fig. 4 is a diagrammatic presentation of the sites at which HF is cleaved to form HFf. An initial cleavage at site 1 within a disulfidelinked portion of the molecule produces $\mathrm{HFa}$. This cleavage is made by either $\mathrm{HFa}$ (autodigestion) $(1,6$, $12,13)$ or by kallikrein $(1,4,14)$. Subsequently, cleavage occurs at site 2 , to generate the $30,000-\mathrm{mol}$ wt form of HFf followed by a final cleavage at site 3 to generate the 28,500-mol wt form. Both of these cleavages are proposed to occur to the left of the initial cleavage at site $1(\mathrm{HFa})$. A fourth cleavage, which occurs to the left of site 3 , can occur, and in the absence of cleavage at sites 2 or 3 , will produce the 40,000 dalton active species. Data presented previously (1) indicates that this cleavage is made primarily by $\mathrm{HFa}$. Thus, when $\mathrm{HF}$ is incubated with kallikrein in the presence of an initiating surface (e.g., dextran sulfate) the 40,000-dalton molecule is formed in very minor quantities, if at all (Fig. 1), presumably because of the efficiency of kallikrein cleavage at sites 2 and 3 . However, when $\mathrm{HF}$ is incubated with prekallikrein in the fluid phase (in a plastic tube), the 40,000-dalton enzyme is generated (Fig. 2) (1).

The data presented in Fig. 3 indicate that the 40,000-dalton enzyme and HFf all consist of a heavy chain of 28,000 daltons disulfide linked to light chains. The light chain of the 40,000-dalton enzyme appears to have a molecular weight of 15,000 . It is important to note that a molecular weight of 12,000 was reported (1) for this chain using a nongradient SDS gel electrophoresis system. Molecular weight standards run in both systems generated linear standard curves from which molecular weights of the samples were determined. The difference of molecular weights reported appears to be due to the differing gel systems. A light chain of 2,000 daltons is generated from the left side portion of the HFf position, (Fig. 3.), and is therefore generated from the 30,000 -dalton molecule. A small amount of radiolabeled material is visible at the electrophoretic front of the gel and is generated only from the right portion of the HFf position. No material is present at the front beneath the 40,000-dalton position nor the left portion of the HFf position. Because the material runs at the electrophoretic front, the molecular weight must be $<1,000$ and might represent the light chain associated with the 28,500 -dalton molecule.

Revak et al. (2) have presented a model suggesting that the disulfide bridge area within which activation occurs (site 1) is small and that formation of HFf could occur by cleavage to the left or right of this area. Because HFf has two molecular forms, it is also possible that cleavage occurs at both sites. Cleavage to the right of site 1 would expose a different $\mathrm{N}$-terminus; however, the $\mathrm{N}$-terminal residue exposed by site 1 cleavage is thought to be important for the mechanism of action of serine proteases. It folds into the interior of the protein to form an ion pair with the carboxyl group of the aspartic acid residue adjacent to the active site serine (15) and is critical for the charge-relay system

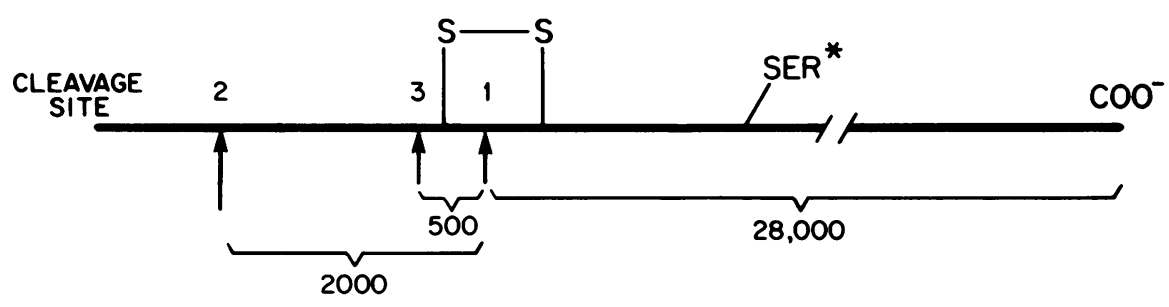

Figure 4 Diagrammatic representation of the band cleavages associated with HF activation. Cleavage at site 1 generates $\mathrm{HFa}$. Cleavage at sites 2 and 3 generates the two forms of $\mathrm{HFf}$. Alternatively, cleavage to the left of site 3 , subsequent to cleavage at site 1 , would generate larger activated fragments such as the 40,000-dalton enzyme. 
(16). Cleavage to the right of site 1 would also require that one of these forms have a molecular weight less than that of the light chain of $\mathrm{HFa}$. Because all the forms of activated $\mathrm{HF}$ possess a 28,000 -dalton disulfide linked chain (1), and HFa is the precursor of the various active fragmentation products, we propose that the sites of cleavage that produce both active species of HFf as well as the 40,000-dalton enzyme are located to the left of site 1 and outside of the disulfide-linked portion of the molecule at which site 1 cleavage occurs. Revak et al. (10) have also suggested that HF cleavage may proceed nonsequentially to form either $\mathrm{HFa}$ by one mechanism (site 1 cleavage) or HFf by a separate mechanism involving cleavage external to the initial disulfide. The active forms resulting were designated $\alpha$ and $\beta$. Because HF cleavage appears to be sequential, and the various fragmentation products are not significant coagulation factors $(2,5,10)$ we feel that $\mathrm{HFa}$ should be used to designate the active coagulant enzyme and HFf be reserved for low molecular weight species $(28,500-30,000)$, which function to convert prekallikrein to kallikrein (5) and thereby lead to kinin formation. Other intermediately sized fragments are not seen when normal plasma is activated because the process is kallikrein-dependent, but may be important when prekallikrein-deficient plasma is activated.

\section{REFERENCES}

1. Dunn, J. T., M. Silverberg, and A. P. Kaplan. 1982. The cleavage and formation of Activated Human Hageman Factor by autodigestion and by kallikrein. J. Biol. Chem. 257: 1779-1781.

2. Revak, S. D., C. G. Cochrane, and J. H. Griffin. 1977. The binding and cleavage characteristics of human Hageman factor during contact activation: a comparison of normal plasma with plasma deficient in factor XI, prekallikrein, and high-molecular weight kininogen. $J$. Clin. Invest. 59: 1157-1175.

3. Kaplan, A. P., and K. F. Austen. 1971. A prealbumin activator of prekallikrein II. Derivation of activators of prekallikrein from activated Hageman factor by digestion with plasmin; J. Exp. Med. 133: 696-712.

4. Cochrane, C. G., S. D. Revak, and K. D. Wuepper. 1973. Activation of Hageman factor in solid and fluid phases. A critical role for kallikrein. J. Exp. Med. 38: 1564-1538.

5. Kaplan, A. P., and K. F. Austen. 1971. A prealbumin activator of prekallikrein. J. Immunol. 105: 802-811.

6. Silverberg, M., J. T. Dunn, L. Garen, and A. P. Kaplan. 1980. Autoactivation of human Hageman factor: demonstration utilizing a synthetic substrate. J. Biol. Chem. 255: 7281-7286.

7. Mandle, R., and A. P. Kaplan. 1977. Hageman factor substrates. Human plasma prekallikrein: mechanism of activation by Hageman factor and participation in Hageman factor-dependent fibrinolysis. J. Biol. Chem. 252: 6097-6104.

8. Lowry, O. H., N. J. Rosenbrough, A. L. Farr, and R. J. Randall. 1951. Protein measurement with the Folin phenol reagent. J. Biol. Chem. 193: 265-275.

9. Laemmli, V. K. 1970 . Cleavage of structural proteins during the assembly of the head of bacteriophage T4. Nature (Lond.). 227: 680-685.

10. Revak, S. D., C. G. Cochrane, B. N. Bouma, and J. H. Griffin. 1978. Surface and fluid phase activities of two forms of activated Hageman factor produced during contact activation of plasma. J. Exp. Med. 147: 719-729.

11. Fujikawa, K., K. Kurachi, and E. W. Davie. 1977. Characterization of Bovine Factor XIIa (Activated Hageman factor). Biochemistry. 16: 4182-4188.

12. Wiggins, R. C., and C. G. Cochrane. 1979. The autoactivation of rabbit Hageman factor. J. Exp. Med. 150: 1122-1133.

13. Miller, G., M. Silverberg, and A. P. Kaplan. 1980. Autoactivation of human Hageman factor (Factor XII). Biochem. Biophys. Res. Commun. 92: 803-810.

14. Fujikawa, K., R. L. Heimark, K. Kurachi, and E. W. Davie. 1980. Activation of Bovine Factor XII (Hageman factor) by plasma kallikrein. Biochemistry. 19: 13221330.

15. Sigler, P. B., D. M. Blow, B. W. Matthews, and R. Henderson. 1980. Structure of crystalline $\alpha$-chymotrypsin II. A preliminary report including a hypothesis for the activation mechanism. J. Mol. Biol. 35: 143-164.

16. Shotton, D. M., and H. C. Watson. 1970. Three dimensional structure of tosyl elastase. Nature (Lond.). 225: 811-816. 